\title{
The revelation effect: Moderating influences of encoding conditions and type of recognition test
}

\author{
Neil W. Mulligan \\ University of North Carolina, Chapel Hill, North Carolina
}

\begin{abstract}
In the revelation effect, slowly revealing a test item immediately prior to a recognition judgment increases the probability of an "old" response. Extant accounts imply that the revelation effect occurs for familiarity-based judgments but not for those involving recall or recollection. To assess this implication, the contribution of recollection was varied by encoding conditions and memory tests. Two manipulations of recollection, semantic encoding and context-item integration, both reduced the revelation effect on a standard recognition test. For the same encoding conditions, a perceptually driven memory test (rhyme recognition) exhibited no revelation effect whatsoever. In a second experiment, the rhyme recognition test exhibited a revelation effect under impoverished encoding conditions. The results document important limits of the revelation effect produced by both encoding and test conditions. Furthermore, the recollection-based limitations of the revelation effect are not restricted to conditions of enhanced semantic encoding.
\end{abstract}

Memory researchers frequently analyze memory errors and misattributions to elucidate normal memory processes. One such misattribution is the revelation effect (Watkins \& Peynircioğlu, 1990), which contrasts two retrieval conditions. In the revelation condition, a test item is slowly revealed or presented as an anagram to be solved, after which the participant makes an old-new recognition judgment. This is contrasted with a typical test condition, in which the test item is presented in its normal form. The revealed items elicit more "old" responses independent of the actual old-new status.

The revelation effect is quite general. It occurs for solving anagrams (Watkins \& Peynircioğlu, 1990; Mulligan \& Lozito, 2006), revealing words letter by letter (e.g., LeCompte, 1995), identifying perceptually degraded words (Luo, 1993) or reading rotated or inverted words (e.g., Watkins \& Peynircioğlu, 1990). The effect occurs with a variety of materials including words, numbers, pictures and faces (e.g., Bornstein \& Wilson, 2004; Luo, 1993). Several memory tests exhibit the effect including recognition, frequency judgments and judgments of autobiographical memory (Bernstein, Whittlesea, \& Loftus, 2002). Finally, preceding the recognition decision with an unrelated task (e.g., an anagram of a different word, a math problem) can also produce the revelation effect (e.g., Niewiadomski \& Hockley, 2001; Westerman \& Greene, 1998; cf. Leynes et al., 2005; Verde \& Rotello, 2004). Indeed, the generality of the effect has prompted the observation that attempts to find its limiting conditions have succeeded primarily in demonstrating the effect's gener- ality (Westerman \& Greene, 1998). As such, it is critical to document limiting conditions of the effect to constrain theorizing (Bornstein \& Wilson, 2004).

The revelation effect has been analyzed in terms of the dual-process model of recognition memory (e.g., Azimian-Faridani \& Wilding, 2004; Cameron \& Hockley, 2000), a theory which differentiates between an ahistorical feeling of familiarity and a recall-like recollection process (Yonelinas, 2002). Extant accounts imply that the revelation effect is linked to familiarity-based judgments but not to recollection. These accounts are of several different types. First, some of the accounts argue that revelation influences familiarity values directly, enhancing the perceived familiarity of both new and old items (e.g., Leynes et al., 2005; Luo, 1993; Westerman \& Greene, 1998). A related account argues that revelation affects the evaluation and attribution of familiarity (e.g., Bernstein et al., 2002). Other hypotheses argue that revelation induces a liberal criterion shift relative to the control condition thus increasing "old" responding (e.g., Niewiadomski \& Hockley, 2001; Verde \& Rotello, 2004). Finally, Hicks and Marsh (1998), upon finding a negative effect of revelation in forced choice recognition, argued that revelation actually decreases familiarity but simultaneously induces a liberal criterion shift, resulting in the familiar revelation effect for standard yes-no recognition.

Within the dual-process framework, all of the foregoing accounts link the revelation effect to familiarity. The former accounts do so directly, arguing that revelation directly affects familiarity or the attribution of familiarity.

N.W.Mulligan,nmulligan@unc.edu 
The criterion-shift accounts do so indirectly. In terms of the dual-process account, criterion-mediated decisions are assumed to be relevant only for familiarity-based responding (Azimian-Faridani \& Wilding, 2004; Leynes et al., 2005; Yonelinas, 2002). Despite differences between the accounts, all of these accounts imply that revelation influences familiarity-based memory decisions but not recollection (Azimian-Faridani \& Wilding, 2004; Cameron \& Hockley, 2000). I refer to this as the recollection-limitation hypothesis.

Accordingly, memory judgments based primarily on recollection should not exhibit the revelation effect. There is some evidence in favor of this view. Cameron \& Hockley (2000; Westerman, 2000) found no revelation effect on associative recognition, a task in which participants study word pairs (e.g., A-B, C-D, etc.) and are later tested with intact targets (e.g., A-B) or lures made from rearranged study pairs (e.g., A-D). Nonspecific familiarity is not particularly useful here because the individual test words are all familiar. Consequently, this task relies heavily on recall of the exact study pairing. The lack of a revelation effect on this task is consistent with the notion that revelation influences familiarity-based but not recollection-based memory judgments. Westerman (2000) reported a similar finding using another test with a heavy reliance on recollection, the plurality recognition test (which requires distinguishing between studied items and lures differing in plurality).

The present study evaluates the recollection-limitation hypothesis by examining different encoding conditions and memory tests, including standard recognition. Only one prior study has assessed this notion with standard recognition. Landau (2001) varied study duration in an attempt to alter the contribution of recollection and familiarity, the assumption being that longer (vs. shorter) study duration increases the contribution of recollection at test and thus diminishes the revelation effect. Although the results were consistent with this prediction, study duration may not be the optimal encoding manipulation because it may affect both familiarity and recollection (Yonelinas, 2002, p. 459). It is unclear if this manipulation differentially influenced recollection, a necessary condition for assessing the recollection-limitation hypothesis. The present study used the levels-of-processing (LOP) manipulation because of its clear effects on recall and recollection (Yonelinas, 2002). According to the recollection-limitation hypothesis, the revelation effect should be reduced for semantic compared to nonsemantic encoding because the influence of recollection is greater in the former condition.

Within the dual-process literature, there is a very strong association between enhanced semantic encoding and enhanced recollection (especially for verbal materials). Consequently, it is natural to wonder if the effects of enhanced recollection are equivalent to the effects of enhanced semantic encoding, or if enhanced recollection brought about by other means would similarly moderate the revelation effect. This is addressed in two ways in the present experiment. First, we used a classic LOP manipulation (Craik \& Tulving, 1975) in which each study word is preceded by a yes-no orienting question, half of the which were semantic (e.g., Is this a type of tool? DRILL) and half nonsemantic, relating to the word's sound (e.g., Does this word rhyme with spill? DRILL). Furthermore, half of the questions had a correct answer of yes and half an answer of no. The semantic-nonsemantic comparison constitutes the typical LOP manipulation. Question answer also affects memory; yes items produce better recognition and recall than no items (e.g., Craik \& Tulving, 1975). This effect is distinct from the effect of semantic processing, occurring in both the semantic and nonsemantic conditions. Craik and Tulving argued that the yes questions enhance context integration between the word and its question context. That is, in the yes condition, it is easier to retrieve information about the study (e.g., question) context, providing an additional basis for accurate "old" decisions. In terms of the dual process model, the retrieval of contextual information is a defining characteristic of recollection. Thus, the recollection-limitation hypothesis predicts that the revelation effect will be reduced for yes- compared to no-items, allowing an assessment of this hypothesis based on a second manipulation, one orthogonal to the manipulation of semantic encoding.

The present study assessed the relationship between semantic encoding, recollection and the revelation effect in a second way, using a recognition test with little reliance on semantics, the rhyme recognition test. Morris, Bransford, and Franks (1977) developed this task to show that semantic encoding does not always produce superior memory. In rhyme recognition, participants determine whether test words rhyme with studied words. In this task, phonetically encoded words are better remembered than semantically encoded words (a reversal of the typical LOP effect). Thus, the rhyme recognition test differs from standard assessments of episodic memory in that it appears to be perceptually rather than conceptually driven. The rhyme recognition test was used in the present study to determine if the revelation effect is found on a test that has little reliance on semantics. Given the close connection between semantic processing and enhanced recollection, it might be expected that a nonsemantic recognition test would exhibit a revelation effect just as nonsemantic encoding is expected to produce a revelation effect in standard recognition. In contrast, recent research indicates that rhyme recognition is negatively effected by divided attention during retrieval, a marker for recollection-based retrieval (Lozito \& Mulligan, 2006). Lozito and Mulligan argued that rhyme recognition is typically dominated by recall processes rather than familiarity. According to the recollection-limitation hypothesis, recall-based memory judgments should not produce a revelation effect.

\section{EXPERIMENT 1}

\section{Method}

Participants. The participants were 80 undergraduates at the University of North Carolina.

Design and Materials. Encoding condition (semantic vs. phonetic), question type (yes vs. no) and test condition (intact vs. revealed) were manipulated within-subjects. Memory test (standard vs. rhyme recognition) was varied between subjects. The critical words were 96 common five-letter words. Four orienting questions were constructed for each word. Two of the questions emphasized word sound, 
one with a yes answer (phonetic-yes, PY) the other with an answer of no (phonetic-no, PN). The other two questions emphasized word meaning, one with a yes answer (semantic-yes, SY) and the other a no answer (semantic-no, SN). Each critical word rhymed with at least two other words and bore no obvious semantic or phonetic relation to the other critical words. Four additional words with similar characteristics were used as primacy (two) and recency (two) buffers.

The critical items were randomly divided into two sets for the oldnew counterbalance. Each set was used to create four versions of the study list, counterbalancing items across the encoding-question categories (PY, PN, SY, and SN). The standard recognition test consisted of all 96 critical items (48 old and 48 new). The rhyme recognition test consisted of 96 words that rhymed with one, and only one, of the critical items. None of the test words appeared in any of the orienting questions. Half of the test items of each type were presented in the intact condition and half in the revealed condition (the test items were randomly intermixed). Two versions of the recognition tests were created to counterbalance items over test conditions.

Procedure. The experiment consisted of a study phase, a distractor task, and the recognition test. Participants were informed that they would hear a list of words and that they should try to remember the words for a later (unspecified) memory test. On each study trial, the yes-no question appeared on the computer screen $3 \mathrm{sec}$ before the word was presented over the computer speakers. Upon response, the screen blanked for $500 \mathrm{msec}$ and then the next trial began. The study phase was followed by a 3-min arithmetic distractor task.

On the standard recognition test, half of the test words were initially presented as anagrams (the revelation condition). The anagram was presented with a number below each letter indicating the position of the letter in the anagram's solution (e.g., a " 1 " indicating the first letter of the solution) to ensure that the anagrams would be solved. The participant wrote the solution on an answer sheet and then hit the space bar to proceed to the recognition decision. The test item was then displayed in its normal form and the participant indicated the recognition decision by typing $\mathrm{O}$ (for old) or $N$ (for new). On the intact trials, the test item was presented in its normal form for a recognition decision.

In the rhyme recognition test, participants indicated whether the test word rhymed with a word that was heard in the study list. In this case, the "old" test items rhymed with studied words whereas the "new" items rhymed with unpresented critical items. Otherwise, the revelation and intact trials proceeded as in the standard recognition test. ${ }^{1}$

\section{Results and Discussion}

Standard recognition. The test results are presented in Table 1. A preliminary analysis verified the expected revelation effect. Hits (averaged over encoding conditions) and false alarms were analyzed using study status (old vs. new) and test condition (intact vs. revealed) as factors. The effect of test condition indicates that solving an anagram prior to recognition increased the "old" responses

Table 1

Experiment 1: Mean Hit and False Alarm (FA) Rates As a Function of Encoding, Question, and Memory Test Conditions

\begin{tabular}{lllllll}
\hline & \multicolumn{5}{c}{ Hits } \\
\cline { 2 - 5 } Condition & PN & PY & SN & SY & Average & FAs \\
\hline Standard Recognition Test & & & & & \\
$\quad$ Intact & .50 & .66 & .67 & .87 & .67 & .19 \\
$\quad$ Revealed & .65 & .72 & .74 & .83 & .73 & .27 \\
Rhyme Recognition Test & & & & & & \\
$\quad$ Intact & .53 & .69 & .51 & .57 & .57 & .30 \\
$\quad$ Revealed & .55 & .63 & .49 & .54 & .55 & .29 \\
\hline
\end{tabular}

Note-PN, phonetic-no; PY, phonetic-yes; SN, semantic-no; SY, semantic-yes. (i.e., the revelation effect was obtained) $[F(1,57)=54.32$, $\left.M S_{\mathrm{e}}=0.004, \alpha=.05\right]$; the interaction was nonsignificant $(F<1)$. Next, the hit rates were analyzed with a 2 (encoding condition) $\times 2$ (question type) $\times 2$ (test condition) ANOVA. ${ }^{2}$ There were five significant effects. First, the effect of encoding condition $\left[F(1,47)=33.39, M S_{\mathrm{e}}=\right.$ 0.062], demonstrates the typical LOP effect. Second, the effect of question type $\left[F(1,47)=59.98, M S_{\mathrm{e}}=0.028\right]$, indicates that yes-items produce better memory than noitems (consistent with prior results, Craik \& Tulving, 1975; Lozito \& Mulligan, 2006). The last three effects were those of test condition $\left[F(1,47)=17.64, M S_{\mathrm{e}}=0.021\right]$, the testcondition-by-encoding-condition interaction $[F(1,47)=$ 6.91, $\left.M S_{\mathrm{e}}=0.029\right]$, and the test-condition-by-questiontype interaction $\left[F(1,47)=5.23, M S_{\mathrm{e}}=0.044\right]$. The interactions indicate that the revelation effect was moderated by encoding condition and by question type (the remaining interactions were nonsignificant, $F_{\mathrm{S}}<1$ ). Additional analyses clarify the interactions. First, the revelation effect was significant in the phonetic condition (intact $=.58$, revealed $=.68)\left[F(1,47)=18.28, M S_{\mathrm{e}}=0.030\right]$, but not in the semantic condition (intact $=.77$, revealed $=.78$; $F<1)$. Likewise, the revelation effect was significant for no questions (intact $=.58$, revealed $=.69)[F(1,47)=$ $\left.19.62, M S_{\mathrm{e}}=0.030\right]$, but not for yes questions (intact $=$ .76 , revealed $=.78 ; F<1)$.

The critical results are the test condition interactions. First, the results indicate that semantic elaboration, the standard encoding manipulation for enhancing recollection, reduces the revelation effect. Second, question type provides an alternate means to vary recollection, one that is independent of semantic encoding (Craik \& Tulving, 1975). This manipulation also moderated the revelation effect, with a reduced revelation effect in the yes condition, the condition producing greater context integration and recollection. These results are consistent with the idea that revelation affects familiarity-based recognition but not recollection. Perhaps not surprisingly, the SY condition (i.e., the combination of the conditions expected to enhance recollection) produced the starkest result, with a small (nonsignificant) reversal of the usual revelation effect.

Conditions enhancing recollection tend to produce higher recognition accuracy, raising the concern that the lack of a revelation effect under semantic encoding or yes questions is a ceiling artifact. To address this issue, the recognition data were subjected to a median split on average recognition accuracy. Hit rates were reanalyzed adding performance level as a factor. This factor did not significantly interact with test condition (or any other factor), indicating that the revelation effect was not moderated by performance level. The average revelation effect on hits was .06 at both high and low performance levels. In the semantic condition, the revelation effect averaged .01 and .02 in the high and low groups, respectively. For the yes questions, the corresponding averages were .05 and -.02 . Thus, there is no indication that the revelation effect emerged in the semantic or yes conditions as performance levels decreased, arguing against a ceiling artifact.

The signal-detection measures of sensitivity $\left(d^{\prime}\right)$ and criterion $(c)$ are presented in Table 2. Averaged across encod- 
ing conditions, test condition had no effect on $d^{\prime}$ accuracy $(t=1.06)$ but did affect $c[t(47)=6.37]$, indicating a more liberal criterion placement in the revelation condition. ${ }^{3}$ These results are consistent with the finding that revelation produced equivalent increases in hits and false alarms.

Rhyme recognition. The analysis of hits and false alarms indicated no effect of test condition $(F<1)$. Thus, there was no evidence of a revelation effect in rhyme recognition. Next, the hit rates were analyzed with a 2 (encoding condition) $\times 2$ (question type) $\times 2$ (test condition) ANOVA. This analysis verifies that the present results replicate prior research and that rhyme recognition is as a perceptually driven test. Specifically, there was a main effect of encoding condition $\left[F(1,31)=4.67, M S_{\mathrm{e}}=0.070\right]$, indicating higher hit rates in the phonetic than semantic condition (i.e., the reversed LOP effect). The main effect of question type was also significant $\left[F(1,31)=8.18, M S_{\mathrm{e}}=0.060\right]$, indicating higher hit rates for yes than no items. Both of these results are consistent with prior findings (e.g., Lozito \& Mulligan, 2006; Morris et al., 1977). No other results were significant ( $p \mathrm{~s}>.15)$. Critically, the effects involving test condition were nonsignificant $\left(F_{\mathbf{s}}<1\right)$. Unlike standard recognition, rhyme recognition was unaffected by the revelation manipulation regardless of the encoding conditions. Analysis of the signal-detection measures (Table 2) indicate no effect of test condition on $d^{\prime}$ or $c(t \mathrm{~s}<1.1)$.

The lack of a revelation effect in rhyme recognition represents one of the few limiting conditions of the revelation effect in episodic memory. This result is consistent with the notion that rhyme recognition requires recollection, that is, retrieval of specific details from the study episode. In particular, it seems likely that this test typically elicits attempts to retrieve the studied word given the rhyming word as a cue, regardless of the encoding condition. In this sense, the rhyme recognition test may routinely rely on recall processes. This is consistent with the recent observation that rhyme recognition is affected by divided attention during test, an indicator of recollection-dependent retrieval (Lozito \& Mulligan, 2006).

\section{EXPERIMENT 2}

Cameron and Hockley (2000) provide a similar analysis of associative recognition, arguing that the task routinely relies on recall processes and thus fails to exhibit a revelation effect. However, Cameron and Hockley argued, if

Table 2

Experiment 1: Sensitivity $\left(d^{\prime}\right)$ and Criterion (c) As a Function of Memory Test Condition

\begin{tabular}{lcc}
\multicolumn{3}{c}{ Function of Memory Test Condition } \\
\hline Condition & $d^{\prime}$ & $c$ \\
\hline Standard Recognition Test & & \\
Intact & 1.48 & .24 \\
Revealed & 1.38 & .01 \\
Rhyme Recognition Test & & \\
Intact & .86 & .22 \\
Revealed & .85 & .32 \\
\hline
\end{tabular}

Note $-d^{\prime}$ and $c$ based on average hit rates averaged over encoding conditions.
Table 3

Experiment 2: Mean Hit Rate, False Alarm (FA) Rate, $d^{\prime}$, and $c$ As a Function of Memory Test Condition

\begin{tabular}{lcccc}
\hline Condition & Hits & FAs & $d^{\prime}$ & $c$ \\
\hline Intact & .49 & .35 & .51 & .23 \\
Revealed & .53 & .41 & .47 & .09 \\
\hline
\end{tabular}

encoding were sufficiently impoverished then recollection would be less likely, making it possible to observe a revelation effect. When word pairs were presented for very brief study durations (500 $\mathrm{msec})$, associative recognition exhibited the revelation effect. Rhyme recognition might behave likewise if encoding were similarly impoverished. Experiment 2 used short study presentations and no orienting questions to produce this situation. ${ }^{4}$

\section{Method}

Participants. Thirty-two undergraduates at the University of North Carolina participated.

Design, Materials, and Procedure. This experiment was identical to the rhyme recognition condition in Experiment 1 except that study words were presented every $600 \mathrm{msec}$ and no orienting questions were presented. Prior to the beginning of the study lists, participants were warned about the rapid presentation of the words.

\section{Results and Discussion}

Experiment 2 succeeded in producing impoverished encoding (performance was substantially worse than in Experiment 1 ). Hits and false alarms (Table 3 ) were submitted to a 2 (study status) $\times 2$ (test condition) ANOVA, revealing an effect of test condition $\left[F(1,31)=7.34, M S_{\mathrm{e}}=0.012\right]$. The proportion of "old" responses was greater in the revealed than intact condition (the interaction was nonsignificant, $F<1$ ). Consistent with this analysis, revelation had no effect on $d^{\prime}, t<|1|$, but significantly reduced $c[t(31)=$ 2.10]. In contrast to Experiment 1, the present experiment demonstrates a revelation effect for rhyme recognition. Consistent with the logic of Cameron and Hockley (2000), reducing the likelihood (or utility) of recall on the rhyme recognition test induces a revelation effect.

\section{GENERAL DISCUSSION}

The revelation effect has proven to be a quite general memory phenomenon, making it critical to delineate the limits of the effect (Bornstein \& Wilson, 2004). The present experiments demonstrate some moderating factors. In standard recognition, semantic encoding and greater item-context integration (as afforded by the yes questions) both limit the revelation effect. These results are consistent with the idea that enhanced recollection diminishes the size of the revelation effect. Furthermore, this result is not restricted to semantic encoding, which is the standard manipulation of recollection. Rather, question type, whose effects occur for semantic and nonsemantic conditions, also moderates the revelation effect. Yes-items increase item-context integration, enhancing recall and recognition (via enhanced recollection) (Craik \& Tulving, 1975; Lozito \& Mulligan, 2006) and so were expected to diminish the revelation effect. 
Experiment 1 also demonstrated that the type of recognition test can limit the revelation effect. Under encoding conditions that produced a revelation effect in standard recognition, rhyme recognition exhibited no revelation effect at all. This is likely because rhyme recognition typically relies on recall processes rather than familiarity. Thus, like associative and plurality recognition (Cameron \& Hockley, 2000; Westerman, 2000), the test of rhyme recognition failed to exhibit a revelation effect under typical encoding conditions. Also, like associative recognition, impoverished encoding conditions causes the revelation effect to emerge. This is critical because it demonstrates that a revelation effect can be found on a perceptually driven task, a task with little reliance on semantic encoding. This converges with the results of standard recognition in distinguishing the effects of enhanced semantic encoding from the effects of recollection.

As described in the introduction, the present experiments were designed to test the common dual-process implication that revelation exerts its effects on familiarity-based responding (e.g., Azimian-Faridani \& Wilding, 2004; Cameron \& Hockley, 2000), rather than to make a finegrained distinction between accounts proposing direct changes to familiarity vs. criterion shift. However, when revelation influenced performance, it influenced $c$ (a standard measure of criterion placement) rather than $d^{\prime}$ (discriminability), a result consistent with criterion-shift accounts (Verde \& Rotello, 2004).

More generally, the present results indicate that encoding manipulations that enhance recollection can limit the revelation effect for old items in a standard recognition test (see also Landau, 2001). This complements results with associative, plurality and rhyme recognition. In the former case, encoding conditions vary the extent of recollection-based responding within a single test. In the latter case, retrieval conditions vary the extent of recollection-based responding across tests. Both strategies have now demonstrated limitations on the revelation effect consistent with the recollection-limitation hypothesis.

\section{AUTHOR NOTE}

Correspondence concerning this article should be addressed to N. W. Mulligan, Department of Psychology, University of North Carolina, Chapel Hill, NC 27599-3270 (e-mail: nmulligan@unc.edu).

\section{REFERENCES}

Azimian-Faridani, N., \& Wilding, E. L. (2004). An event-related potential study of the revelation effect. Psychonomic Bulletin \& Review, 11, 926-931.

Bernstein, D. M., Whittlesea, B. W. A., \& Loftus, E. F. (2002). Increasing confidence in remote autobiographical memory and general knowledge: Extensions of the revelation effect. Memory \& Cognition, 30, 432-438.

Bornstein, B. H., \& WiLson, J. R. (2004). Extending the revelation effect to faces: Haven't we met before? Memory, 12, 140-146.

Cameron, T. E., \& Hockley, W. E. (2000). The revelation effect for item and associative recognition: Familiarity versus recollection. Memory \& Cognition, 28, 176-183.

Craik, F. I. M., \& Tulving, E. (1975). Depth of processing and retention of words in episodic memory. Journal of Experimental Psychology: General, 104, 268-294.
Hicks, J. L., \& MARSh, R. L. (1998). A decrement-to-familiarity interpretation of the revelation effect from forced-choice tests of recognition memory. Journal of Experimental Psychology: Learning, Memory, \& Cognition, 24, 1105-1120.

LANDAU, J. D. (2001). Altering the balance of recollection and familiarity influences the revelation effect. American Journal of Psychology, 114, 425-437.

LeCompte, D. (1995). Recollective experience in the revelation effect: Separating the contributions of recollection and familiarity. Memory \& Cognition, 23, 324-334.

Leynes, P. A., Landau, J., Walker, J., \& Addante, R. J. (2005). Event-related potential evidence for multiple causes of the revelation effect. Consciousness \& Cognition, 14, 327-350.

Lozito, J. P., \& Mulligan, N. W. (2006). Exploring the role of attention during memory retrieval: Effects of semantic encoding and divided attention. Memory \& Cognition, 34, 986-998.

Luo, C. R. (1993). Enhanced feeling of recognition: Effects of identifying and manipulating test items on recognition memory. Journal of Experimental Psychology: Learning, Memory, \& Cognition, 19, 405-413.

Morris, C. D., Bransford, J. D., \& Franks, J. J. (1977). Levels of processing versus transfer appropriate processing. Journal of Verbal Learning \& Verbal Behavior, 16, 519-533.

Mulligan, N. W., \& Lozito, J. P. (2006). An asymmetry between memory encoding and retrieval: Revelation, generation, and transferappropriate processing. Psychological Science, 17, 7-11.

NieWIAdomski, M. W., \& HockLey, W. E. (2001). Interrupting recognition memory: Tests of familiarity-based accounts of the revelation effect. Memory \& Cognition, 29, 1130-1138.

Verde, M. F., \& Rotello, C. M. (2004). ROC curves show that the revelation effect is not a single phenomenon. Psychonomic Bulletin \& Review, 11, 560-566.

Watkins, M. J., \& PeynircioğLu, Z. F. (1990). The revelation effect: When disguising test items induces recognition. Journal of Experimental Psychology: Learning, Memory, \& Cognition, 16, 1012-1020.

Westerman, D. L. (2000). Recollection-based recognition eliminates the revelation effect in memory. Memory \& Cognition, 28, 167-175.

Westerman, D. L., \& Greene, R. L. (1998). The revelation that the revelation effect is not due to revelation. Journal of Experimental Psychology: Learning, Memory \& Cognition, 24, 377-386.

Yonelinas, A. P. (2002). The nature of recollection and familiarity: A review of 30 years of research. Journal of Memory \& Language, 46, 441-517.

\section{NOTES}

1. Our use of the standard revelation manipulation mandated that test items be presented visually whereas the study items were presented aurally. Pilot research indicated that the reversed LOP effect on rhyme recognition (i.e., the signature of this task's nonsemantic nature) is more likely to be obtained with auditory study presentation.

2. Differences in hit rates across encoding conditions or question types can be interpreted as accuracy differences because the hit rates are relative to a common FA rate. This is not the case for test conditions which are relative to different FA rates.

3 . The analyses of $d^{\prime}$ and $c$ focused only on test condition because the encoding conditions share a common false alarm rate. In the assessment of encoding factors (and their interactions) the common false alarm rate cancels out, leaving an analysis of based on $z$-transformed hit rates. These analyses produce results redundant with the analysis of the untransformed hit rates reported in the body of the text.

4. As noted earlier, study duration appears to affect both recollection and familiarity in standard recognition, although there is no comparable research on rhyme recognition. In the present experiment, the combination of the elimination of the study questions (a potent source of contextual encoding) and reduced study time presumably impairs recollection more than familiarity. At the very least, encoding should be sufficiently impoverished to determine if a revelation effect can be found in rhyme recognition under any circumstances.

(Manuscript received June 8, 2006; revision accepted for publication November $14,2006$. 